\title{
Cardioversão Elétrica da Fibrilação Atrial na emergência: o panorama dos Novos Anticoagulantes Orais versus Anticoagulantes Antagonistas da Vitamina $\mathrm{K}$
}

\author{
Electrical Cardioversion of Atrial Fibrillation in emergency: the panorama of the New Direct \\ Oral Anticoagulants versus Vitamin K Antagonists Anticoagulants
}

Cardioversión Eléctrica de La Fibrilación Auricular em urgencias: el panorama del Nuevos Anticoagulantes Orales versus Anticoagulantes Antagonistas de la Vitamina K

Luísa França de Faria ${ }^{1 *}$, Igor Amorim Amaral ${ }^{1}$, Marianne Fonseca Sarto ${ }^{1}$, Murillo Costa Oliveira1, Paloma Souza Matos ${ }^{1}$, Regiane Helena Medeiros Braga ${ }^{1}$, Vitória Aparecida Cunha ${ }^{1}$, Luís Augusto Ferreira ${ }^{1,2}$.

\section{RESUMO}

Objetivo: Contrastar o uso de Antagonistas de Vitamina $\mathrm{K}(\mathrm{AVK})$ versus os Novos Anticoagulantes Orais Diretos (NOAC) na Cardioversão Elétrica (CE) em pacientes portadores de Fibrilação Atrial (FA), no contexto da emergência. Revisão Bibliográfica: Pacientes com FA admitidos no Departamento de emergência possuem como atual tratamento o controle da frequência cardíaca com medicamentos, a cardioversão elétrica ou farmacológica, o uso de ablação por cateter e o controle das complicações tromboembólicas com o uso de anticoagulantes. Diante dessa circunstância, a CE é apropriada como forma de controle do ritmo sinusal agudo, sobretudo em pacientes hemodinamicamente instáveis. Todavia, tal procedimento ocasiona risco aumentado para eventos tromboembólicos, sendo imprescindível a administração de fármacos anticoagulantes previamente ao procedimento. À vista disso, realizou-se a comparação entre os AVKs e os NOACs como terapia principal para a cardioversão elétrica da fibrilação atrial na emergência, em que estes, a despeito de seu menor conhecimento, possuem vantagens pautadas nas melhores qualidade de vida do paciente e na elevada eficácia. Considerações finais: Apesar de resultados prósperos, a literatura científica demanda maior elucidação para instituir amplamente os NOACs como terapia preferencial para a cardioversão da fibrilação atrial.

Palavras-chave: Fibrilação atrial, Cardioversão elétrica, Anticoagulantes, Vitamina K.

\begin{abstract}
Objective: Contrast the use of Vitamin K Antagonists (AVK) versus New Direct Oral Anticoagulants (NOAC) in Electrical Cardioversion (EC) in patients with Atrial Fibrillation (AF), in the emergency context. Literature Review: Patients with AF admitted to the Emergency Department have as current treatment heart rate control with medication, electrical or pharmacological cardioversion, the use of catheter ablation and the control of thromboembolic complications with the use of anticoagulants. Given this circumstance, CE is appropriate as a way to control acute sinus rhythm, especially in hemodynamically unstable patients. However, this procedure entails an increased risk for thromboembolic events, and it is essential to administer anticoagulant drugs prior to the procedure. From this view, a comparison was made between AVKs and NOACs as the main therapy for electrical cardioversion of atrial fibrillation in emergency, in which these, despite their lesser knowledge, have advantages based on better quality of life for the patient and at high efficiency. Final considerations: Despite successful results, the scientific literature requires further elucidation to widely institute NOACs as the preferred therapy for the cardioversion of atrial fibrillation.
\end{abstract}

Key words: Atrial fibrillation, Electric countershock, Anticoagulants, Vitamin K.

${ }_{1}^{1}$ Pontifícia Universidade Católica de Minas Gerais, Betim - MG. *E-mail: luisafariaa@gmail.com

2 Hospital da Unimed, Belo Horizonte - MG.

SUBMETIDO EM: 7/2021

PUBLICADO EM: 7/2021 


\section{RESUMEN}

Objetivo: Contrastar el uso de Antagonistas de la Vitamina K (AVK) versus Nuevos Anticoagulantes Orales Directos (NOAC) en la Cardioversión Eléctrica (CE) en pacientes con Fibrilación Auricular (FA) en el contexto de urgencias. Revisión de la literatura: Los patientes con FA ingressados en Urgencias tienen como tratamiento actual el control de la frecuencia cardíaca con medicación, cardioversión eléctrica o farmacológica, el uso de ablación con catéter y el control de las complicaciones tromboembólicas con el uso de anticoagulantes. Ante esta circunstancia, la CE es adecuada como forma de controlar el ritmo sinusal agudo, especialmente en pacientes hemodinámicamente inestables. Sin embargo, este procedimiento conlleva un mayor riesgo de eventos tromboembólicos y es fundamental la administración de fármacos anticoagulantes antes del procedimiento. Ante esto, se realizó una comparación entre AVK y NOAC como principal terapia para la cardioversión eléctrica de la fibrilación auricular en urgencias, en la que estos, a pesar de su menor conocimiento, tienen ventajas basadas en una mejor calidad de vida para el paciente y en una alta eficiencia. Consideraciones finales: A pesar de los resultados exitosos, la literatura científica requiere más aclaraciones para instituir ampliamente los NOAC como la terapia preferida para la cardioversión de la fibrilación auricular.

Palabras clave: Fibrilación atrial, Cardioversión eléctrica, Anticoagulantes, Vitamina K.

\section{INTRODUÇÃO}

A Fibrilação Atrial (FA) é um distúrbio cardíaco marcado pela completa desarmonia da atividade elétrica atrial de forma sustentada, resultando em uma taquiarritmia supraventricular com sístole ineficaz. Sua relevância clínica é inquestionável, considerando que a FA é a arritmia cardíaca mais prevalente no mundo, sabendo-se que aproximadamente um a cada quatro adultos desenvolverá FA durante a vida (KJEKSHUS VHR e SCHUSTER P, 2020; JANUARY CT, et al., 2019).

Ademais, os dados divulgados pela American Heart Association (AHA), em 2019, estimam que haja aproximadamente 2,7 milhões de portadores de FA, com estimativas de acometimento entre 0,5 a $3 \%$ da população geral. Algumas evidências supõem que, em 2030, a prevalência de FA na União Européia atinja 14 a 17 milhões de pessoas, sendo que esses valores podem aumentar ainda mais, caso esses indivíduos possuam algum fator de risco associado (KIRCHHOF P, et al., 2016).

A FA destaca-se diante das suas repercussões clínicas, devido à substancial morbidade, o que, além de determinar o aumento dos custos em saúde, promove o aumento da ocorrência de eventos tromboembólicos, com riscos 5 vezes maiores de Acidente Vascular Encefálico (AVE), 3 vezes maiores de Insuficiência Cardíaca (IC) e o dobro de mortalidade em comparação aos pacientes sem FA (TRUJILLO TC, et al., 2019; CATURANO A, et al., 2019; ROZEN G, et al., 2018).

Assim, a FA é um motivo frequente para a admissão no Departamento de Emergência (DE) e é responsável pela ocorrência de 3,9 milhões de atendimentos neste setor e pelo aumento em $16 \%$ nas taxas de hospitalização, tal como salientado no estudo realizado nos Estados Unidos no período de 2007 a 2014 (ROZEN G, et al., 2018).

Partindo disso, com o intuito de prevenir os desfechos tromboembólicos desfavoráveis decorrentes da FA, faz-se necessário o uso de anticoagulação oral com antagonistas da vitamina K (AVKs) e, mais recentemente, com anticoagulantes orais diretos (NOACs), sendo que estes últimos superaram algumas das limitações presentes na primeira classe de medicamentos. Todavia, não há uma consonância entre as atuais diretrizes de FA em relação à preconização da classe farmacológica que deve ser administrada previamente à cardioversão elétrica (GULIZIA MM, et al., 2019).

Dessa forma, este estudo caracteriza-se como uma revisão narrativa de literatura com o objetivo de elucidar a fisiopatologia da formação dos trombos na FA, além de enfatizar, no contexto da emergência, a efetividade dos anticoagulantes antagonistas da vitamina $\mathrm{K}$ versus os novos anticoagulantes orais diretos na cardioversão elétrica dos pacientes portadores de FA. 


\section{REVISÃO BIBLIOGRÁFICA}

\section{Trombogênese na Fibrilação Atrial}

A FA tem sido alvo de discussão por representar a principal fonte emboligênica de origem cardíaca, sendo responsável por $45 \%$ dos eventos tromboembólicos graves como o Tromboembolismo Pulmonar (TEP) e o Acidente Vascular Encefálico (AVE). O perfil epidemiológico dos portadores da FA inclui fatores de risco para eventos tromboembólicos, como idade avançada, principalmente acima de 75 anos, história prévia de AVE, presença de IC, Hipertensão Arterial Sistêmica (HAS), Diabetes Mellitus (DM) e sexo feminino, avaliados através do Escore de CHA2-DS2-VASc (SOCIEDADE BRASILEIRA DE CARDIOLOGIA, 2016). Então, tornase fundamental elucidar os mecanismos envolvidos na trombogênese associada à FA para possibilitar a implantação de terapias preventivas das complicações advindas da persistência e do agravamento deste quadro (SBC, 2016).

Os mecanismos relacionados à fisiopatologia da FA implicam na associação entre três fatores: estruturais, eletrofisiológicos e hemodinâmicos. À vista disso, evidencia-se que a fibrose atrial, a inflamação, o encurtamento do período refratário, os canais iônios e conexinas, e o aumento da pressão intra-atrial promovem dismorfismos genéticos e alterações estruturais atriais culminantes na desorganização da atividade elétrica desta câmara cardíaca (FIGUEIREDO MJO e CINTRA FD, 2021).

Assim, a FA desencadeia um remodelamento elétrico dos componentes normais da eletrofisiologia cardíaca no tecido atrial, por meio da entrada acentuada de cálcio nas células através dos canais iônicos. Concomitantemente, levará a ampla despolarização, com consequente inativação das correntes de cálcio e ativação expressiva das correntes de potássio, caracterizando o encurtamento da duração do potencial de ação e a propagação de múltiplos estímulos originados em focos atriais distintos. O desfecho dessa anormalidade na origem dos impulsos cardíacos provoca a contração incoordenada do músculo atrial, levando à permanência do estágio indeterminado de contração parcial e subsequente função sistólica inadequada desta câmara (KHAN AA e LIP GH, 2019; SBC, 2016).

Ademais, a remodelação cardíaca na FA, principalmente no átrio esquerdo, associadas à circunstância da estase sanguínea, estabelece uma condição pró-trombótica na FA que favorece a trombogênese. Partindo disso, a exploração proteômica permite o entendimento da etiopatogenia do trombo e da trombose e, logo, elucidará os preditivos associados na FA, direcionando para os melhores desfechos (BENTLEY R, et al., 2020). A etiopatogenia da formação tromboembólica na FA é multifatorial. No entanto, todas as causas convergem para alterações da Tríade de Virchow, incluindo a estase sanguínea, a disfunção endotelial e o aumento da trombogenicidade, resultando no estado de hipercoagulabilidade (SBC, 2016; KHAN AA e LIP GYH, 2019).

Ao promover uma sístole atrial ineficaz, aproximadamente 15 a $25 \%$ do volume diastólico final ficará retido nas câmaras atriais, contribuindo para a estase sanguínea e a dilatação atrial a longo prazo, colaborando, ainda, para a formação de coágulos de fibrina que podem se acumular, originando trombos. Acredita-se que a fração de ejeção cardíaca, em condições normais, não depende da sinergia da sístole atrial. Entretanto, ao considerar o paciente crítico, atendido no DE, a ausência da contração atrial estimulará um quadro fisiopatológico de disfunção cardíaca. Ademais, alguns sinais e sintomas são fatores alarmantes de condição cardíaca grave, como a instabilidade hemodinâmica, a IC, a isquemia ou a evidência de pré-excitação (GUTIERREZ C e BLANCHARD DG, 2016; MEAD G, et al., 2017).

Diante disso, evidencia-se a importância do manejo adequado do paciente com FA, a fim de evitar as complicações secundárias relacionadas à arritmia e ao tromboembolismo. Para tal fim, é imprescindível a atualização dos serviços de emergência sobre as drogas e os tratamentos mais eficientes para recuperação precoce da função cardíaca e diminuição do risco de vida (MEAD G, et al., 2017; KHAN AA e LIP GYH, 2019).

\section{Cardioversão Elétrica}

O tratamento da FA possui quatro abordagens principais: o controle da Frequência Cardíaca (FC) com medicamentos, a cardioversão elétrica ou farmacológica, o uso de ablação por cateter e o controle das 
complicações tromboembólicas com o uso de anticoagulantes (CATURANO A, et al., 2019). Nesse sentido, a fim de restabelecer o ritmo sinusal em pacientes com FA com consequente melhora da sintomatologia e da qualidade de vida do paciente, pode-se empreender a cardioversão, através de choques de corrente contínua, ou farmacológica (CATURANO A, et al., 2019).

Segundo Hindricks G, et al. (2021), a cardioversão é apropriada como forma de controle do ritmo sinusal agudo em situações de emergência, ou seja, pacientes com FA e hemodinamicamente instáveis, ou em situações não emergenciais. Nesse primeiro caso, é preferível a cardioversão elétrica (CE) de corrente contínua sincronizada pela capacidade de estabelecer imediatamente o ritmo sinusal, além de ser mais eficaz quando comparada à cardioversão farmacológica.

A CE proporciona a recuperação da contratilidade atrial que, por consequência, pode acarretar no deslocamento de trombos atriais. Assim, evidencia-se que essa conduta promove aumento do risco tromboembólico, já proeminente nessa arritmia, o que salienta a necessidade da administração de terapias anticoagulantes (SULZGRUBER S e NIESSNER A, 2018). Tal fato é sustentado por dados que alegam que a CE apresenta um risco de 5 a $7 \%$ de complicações tromboembólicas. Todavia, nos pacientes em uso de anticoagulação apropriada, o risco desse procedimento reduz para 0,5 a 1,6\% (FREDERIKSEN AS, et al., 2018).

Nessa perspectiva, destaca-se que a anticoagulação previamente à CE pode ser considerada um fator protetor ao paciente. Uma das ferramentas utilizadas para direcionar a decisão no uso de anticoagulantes na FA é o escore CHA2-DS2-VASc que possibilita avaliar o risco de AVE e de sangramento e, consequentemente, selecionar adequadamente os pacientes candidatos a terapia antitrombótica. Esse escore pontua 1 para os fatores de risco insuficiência cardíaca congestiva, hipertensão, diabetes, doença vascular (IAM prévio, doença arterial periférica, placa ateromatosa), idade $\geq 65$ anos e sexo feminino, enquanto a pontuação 2 é reservada para idade $\geq 75$ anos e AVE, totalizando-se 9 pontos (GUTIERREZ C e BLANCHARD DG, 2016).

Esses novos fatores de risco para eventos tromboembólicos permitiram incremento no valor preditivo positivo na detecção de AVE, bem como transparência nas recomendações de anticoagulação. Em decorrência da maior efetividade, essa ferramenta foi incorporada nas diretrizes da Sociedade Europeia de Cardiologia na estratificação do risco de acidentes tromboembólicos nos pacientes com FA e, também se encontra nas recomendações do American College of Cardiology (DOORN SV, et al., 2017; MACHLINECARRION MJ, 2021). No contexto da emergência, esse escore é empregado para nortear a administração de anticoagulantes previamente à cardioversão elétrica (GUTIERREZ C e BLANCHARD DG, 2016; DOORN SV, et al., 2017).

À vista disso, a CE de emergência é recomendada, sobretudo, para pacientes com FA de duração menor que 48 horas, sendo imprescindível a administração prévia de terapias anticoagulantes em pacientes com uma pontuação CHA2-DS2-VASc 2 ou mais em homens ou 3 em mulheres. Levando em consideração a AHA, nos casos hemodinamicamente instáveis, a anticoagulação deve ser iniciada imediatamente e continuada por pelo menos 4 semanas após o procedimento, a menos que haja contraindicação (JANUARY CT, et al., 2019).

Todavia, nos pacientes hemodinamicamente estáveis, uma estratégia dos centros médicos é a observação do indivíduo com medicação para o controle de frequência e da coagulação, sendo a CE aplicada, quando necessária, dentro do tempo limite permitido (CATURANO A, et al., 2019; HINDRICKS G, et al., 2021).

Para mais, nos casos de FA com 48 horas ou mais ou de início desconhecido e independentemente do CHA2-DS2-VASc, faz-se necessária a anticoagulação por pelo menos 3 semanas antes da cardioversão ou um ecocardiograma transesofágico negativo, seguido por 4 semanas de anticoagulação após o procedimento. Além disso, caso o paciente possua risco para AVE com pontuação CHA2-DS2-VASc $\geq 2$, a anticoagulação deve ser mantida por tempo indeterminado (RAGO A, et al., 2019; CATURANO A, et al., 2019; TRUJILLO TC, et al., 2019; BRUNETTI ND, et al., 2018).

No cenário da CE, o fármaco apropriado para a anticoagulação, com o intuito de minimizar o risco de AVE e de sangramento prévio ou posterior ao procedimento, não é padronizado pelas diretrizes de FA. Por isso, 
evidencia-se a possibilidade da utilização dos anticoagulantes orais, podendo ser os antagonistas da vitamina $\mathrm{K}$ ou os novos anticoagulantes orais (MAHAFFEY KW e TURAKHIA MP, 2018).

\section{Anticoagulantes Orais Antagonistas da Vitamina K}

Os anticoagulantes orais antagonistas da vitamina $\mathrm{K}$ (AVKs) foram os pioneiros na anticoagulação de pacientes com FA (CATURANO A, et al., 2019). Levando em consideração as alterações do sistema fisiológico desencadeadas por esses fármacos, verifica-se o comprometimento da produção, pelo fígado, de fatores de coagulação II, VII, IX e X, dependentes da vitamina $\mathrm{K}$ (VK). Tais fatores requerem a gamacarboxilação para sua atuação biológica, sendo a VK o cofator deste processo. À vista disso, os AVKs inibem de forma não competitiva a ação desta vitamina com consequente interrupção das reações de carboxilação dos fatores de coagulação. Ademais, sabe-se que a transformação do fibrinogênio em fibrina insolúvel ocorre por meio da enzima proteolítica trombina, a qual se origina dos fatores dependentes da VK. Acredita-se que os AVK possuem ação protetora devido à diminuição dos substratos pré-requisitos para a formação dos trombos (FAWZY AM e LIP GYH, 2019).

A varfarina é um AVK utilizado durante a cardioversão da $F A$, sobretudo nos pacientes com estenose mitral moderada a grave associada à arritmia ou com válvula mecânica (SULZGRUBER P e NIESSNER A, 2017; MILMAN B e BURNS BD, 2021). Nesses casos, a dosagem do fármaco deve basear-se na farmacocinética e na farmacodinâmica, além da variabilidade individual, sendo determinada pela Razão Normalizada Internacional (RNI) que deve ser mantida entre 2 e 3 (CATURANO A, et al., 2019).

Entretanto, esse fármaco demonstra limitações relacionadas ao início da ação farmacológica e ao estreito intervalo terapêutico, além de necessitar de terapia ponte com heparina de baixo peso molecular (HBPM) (SULZGRUBER P e NIESSNER A, 2017; CATURANO A, et al., 2019).

O período verificado entre a ingestão oral do medicamento e a ação farmacológica pode variar entre 3 até 7 dias devido ao tempo mandatório para a eliminação e/ou o consumo dos fatores de coagulação previamente ativados. Em contraposto, o Tempo de Protrombina (TP) pode-se tornar ampliado rapidamente devido à inibição de fatores de coagulação de curta duração, como o fator VII. Desse modo, embora os AVKs possuam essas barreiras, constata-se que são responsáveis pela redução em $67 \%$ do risco de AVE isquêmico e em $25 \%$ da taxa de mortalidade por causas tromboembólicas nos pacientes com $\mathrm{FA}$, quando comparado à aspirina (CATURANO A, et al., 2019).

No entanto, apesar de mostrarem-se efetivos na proteção dos eventos tromboembólicos, o início vagaroso da ação farmacológica da varfarina consiste em uma grande desvantagem da aplicabilidade desse medicamento na CE, sobretudo a eletiva, já que esse procedimento pode ser delongado, a depender dos níveis de RNI. Consequentemente, esse atraso apresenta uma notável repercussão negativa na qualidade de vida dos pacientes, já que a CE está diretamente relacionada à remissão da sintomatologia da FA. Além disso, a cardioversão rápida proporciona a manutenção do ritmo sinusal a longo prazo e diminui o risco de remissão da FA. Desse modo, acredita-se que os pacientes com CE adiada pela ação farmacológica lenta da varfarina apresentam maior probabilidade de FA paroxística (FREDERIKSEN AS, et al., 2018).

Ademais, esses fármacos possuem reduzido intervalo terapêutico, ou seja, a dose necessária para o efeito almejado é próxima daquela capaz de ocasionar efeitos colaterais significativos ou sinais de intoxicação. Dessa maneira, é imprescindível o monitoramento frequente dos pacientes para manutenção do efeito da anticoagulação terapêutica por meio do $\mathrm{RNI}$, a fim de realizar o ajuste adequado da dose (SULZGRUBER $P$ e NIESSNER A, 2017; CATURANO A, et al., 2019).

Associado a isso, o efeito farmacológico é variável devido à interação dos AVKs com outros medicamentos, como os Anti-Inflamatórios Não Esteroidais (AINEs) que podem provocar hemorragias, o sucralfato que pode alterar a absorção da varfarina e a quinidina que pode aumentar a quantidade de droga livre disponível. Isto posto, evidencia-se que a administração dos antagonistas da vitamina $\mathrm{K}$ apresenta dificuldades clínicas, 0 que pode ser salientado pelo uso de drogas alternativas, como os Novos Anticoagulantes Orais (NOACs) (CATURANO A, et al., 2019; FAWZY AM e LIP GYH, 2019). 


\section{Novos Anticoagulantes Orais}

Os NOACs inibem de forma seletiva apenas um fator da cascata da coagulação, podendo ser a trombina, no caso da dabigatrana, ou o fator $\mathrm{X}$ ativado na rivaroxabana, na apixabana e na edoxabana. Esses fármacos apresentam administração mais fácil e a farmacocinética é previsível, possuindo menores interações alimentares ou medicamentosas. Para mais, esses medicamentos possuem início de ação rápido e bem estabelecido, variando entre uma a quatro horas, quando comparados com os 2 a 7 dias necessários para a varfarina atingir o seu estado estacionário. Além disso, o efeito dos NOACs termina logo após cessar o uso, adicionando-se ainda o benefício de que a resposta é dose previsível. A segurança e a eficácia apresentaram resultados positivos, sabendo-se ainda que a janela terapêutica é maior (CATURANO A, et al., 2019; COPPOLA G, et al., 2019; RENDA G, et al., 2016).

Outro benefício apresentado pelos NOACs é a melhor satisfação do paciente, considerando que não há a necessidade da coleta de sangue obrigatória para a realização da monitorização contínua da faixa de RNI terapêutico, haja vista que esses anticoagulantes não possuem as flutuações na anticoagulação, como ocorre nos AVKs. Logo, a cardioversão não é atrasada quando os níveis de RNI estão anormais (BRUNETTI ND, et al., 2018; GUPTA S, et al., 2019; COPPOLA G, et al., 2019; RENDA G, et al., 2016).

Considerando as vantagens apresentadas pelos anticoagulantes orais, nos últimos anos, esses fármacos se apresentam como uma boa alternativa ao uso dos AVKs. À vista disso, a varfarina, que consistia no anticoagulante oral mais utilizado na FA, vem perdendo espaço para os NOACs por estes apresentarem uma relação risco-benefício não inferior ou, até mesmo, superior, na prevenção de eventos tromboembólicos, além de menor sangramento em contraponto com os AVK. Tal fato é salientado ao evidenciar que os NOACs demonstram reduzir o risco de AVE em pelo menos $60 \%$, enquanto o risco relativo reduziu em mais de $40 \%$ quando comparado aos AVKs (KJEKSHUS VHR e SCHUSTER P, 2020).

Ademais, o uso de NOACs em pacientes com FA pode reduzir ainda mais o risco combinado de AVE e de eventos embólicos em 19\%, bem como o risco de mortalidade por todas as causas em 10\% (RAGO A, et al., 2019; KJEKSHUS VHR e SCHUSTER P, 2020; GUPTA S, et al., 2019).

No cenário emergencial, vale mencionar que os NOACs figuram como fármacos úteis, na medida em que na cardioversão imediata pela instabilidade hemodinâmica ou pela duração da FA de menos de 48 horas, essa nova classe farmacológica permite dispensar a ponte de heparina bem como eliminar a necessidade da troca por outro anticoagulante (RENDA G, et al, 2016).

Dessa forma, é evidente que a utilização dos NOACs não possui nível de segurança inferior quando comparado ao uso dos AVKs, sendo que ainda é acrescido o benefício de dispor de menor risco de AVE e de embolia sistêmica, sugerindo uma eficácia superior dos NOACs (BRUNETTI ND, et al., 2018). Contudo, apesar dessas vantagens, os NOACs ainda carecem de agentes de reversão confiáveis e largamente disponíveis (GUPTA S, et al., 2019).

O resultado de um estudo multinacional, randomizado e controlado, publicado pela Oxford University Press em nome da Sociedade Europeia de Cardiologia, indicou que o uso de apixaban foi consideravelmente mais eficaz quando comparado ao uso dos AVKs ou da heparina, no contexto de cardioversão dos portadores de FA (EZEKOWITZ MD, et al., 2018).

Sendo assim, a comunidade científica demonstra empenho em investigar a eficácia dos NOACs como medida terapêutica consolidada. Perante isto, justifica-se a elevação do uso desses medicamentos na prática clínica, uma vez que diferentes diretrizes, como a da AHA, publicada em 2019, postulam o início da anticoagulação com os NOACs o quanto antes de cada cardioversão em portadores de FA (RAGO A, et al., 2019).

Todavia, vale mencionar que, apesar dos NOACs serem promissores, é preciso individualizar a decisão do uso de acordo com as características de cada paciente, sabendo que os AVKs ainda realizam uma importante função em diversas circunstâncias, inclusive nos casos em que há contraindicações ao uso dos novos anticoagulantes orais (CATURANO A, et al., 2019). 


\section{CONSIDERAÇÕES FINAIS}

Compreende-se que a cardiversão elétrica em FA associado a utilização de NOACs possui melhores desfechos e eficácia na prevenção de eventos tromboembólicos se comparados ao tratamento com uso de AVKs. Sendo assim, é indicado a rápida administração desses fármacos na CE uma vez que seus efeitos são uteis para as repercussões esperadas. Apesar dos resultados promissores, as diretrizes atuais não inserem os NOACs como terapia de escolha, mas como prioridade, fazendo-se necessários novos estudos para difundir mais a utilização desses fármacos e melhorar a qualidade de vida do paciente.

\section{AGRADECIMENTOS}

Agradecemos ao Diretório Acadêmico Horizontal de Medicina da PUC Minas (DAHMP-MG) e à Liga Acadêmica de Medicina de Emergência da Pontifícia Universidade Católica de Minas Gerais (LAMEDE PUCMG) que oportunizaram a produção e a publicação deste estudo.

\section{REFERÊNCIAS}

1. BENTLEY R, et al. Pathophysiological insights into atrial fibrillation: revisiting the electrophysiological substrate, anatomical substrate, and possible insights from proteomics. Cardiovascular Research, 2020; 117(3): e41-e45.

2. BRONCHTEIN AIC. Editorial Revista International Journal of Cardiovascular Sciences - Desafios para Anticoagulação na Fibrilação Atrial, Sociedade de Cardiologia do Estado do Rio de Janeiro (SOCERJ), 2019.

3. BRUNETTI ND, et al. Direct oral anticoagulants compared to vitamin-K antagonists in cardioversion of atrial fibrillation: an updated meta-analysis. J Thromb Thrombolysis, 2018; 45(4): 550-556.

4. CATURANO A, et al. Atrial Fibrillation and Stroke. A review on the use of Vitamin K Antagonists and Novel Oral Anticoagulants. Medicina (Kaunas), 2019; 55(10): 1-11.

5. COPPOLA G, et al. Management of Direct Oral Anticoagulants in Patients with Atrial Fibrillation Undergoing Cardioversion. Medicina, 2019; 55(10): 660-669.

6. DOORN SV, et al. Predictive performance of the CHA2DS2-VASc rule in atrial fibrillation: a systematic review and meta-analysis. Journal of Thrombosis and Haemostasis, 2017; 15(6): 1065-1077.

7. EZEKOWITZ MD, et al. Apixaban compared to heparin/vitamin $\mathrm{K}$ antagonist in patients with atrial fibrillation scheduled for cardioversion: the EMANATE trial. European Heart Journal, 2018; 39: 2959-2971.

8. FAWZY AM, LIP GYH. Pharmacokinetics and Pharmacodynamics of Oral Anticoagulants used in Atrial Fibrillation. Expert Opinion on Drug Metabolism \& Toxicology, 2019; 15(5): 381-398.

9. FIGUEIREDO MJO, CINTRA FD. Fibrilação Atrial (Parte 1): Fisiopatologia, Fatores de Risco e Bases Terapêuticas. Arq. Bras. Cardiol., 2021; 116(1): 129-139.

10. FREDERIKSEN AS, et al. Cardioversion of atrial fibrillation in a real-world setting: non-vitamin $\mathrm{K}$ antagonist oral anticoagulants ensure a fast and safe strategy compared to warfarin. EP Europace, 2018; 20(7): 1078-1085.

11. GULIZIA MM, et al. Management of atrial fibrillation in the emergency room and in the cardiology ward: the BLITZ AF study. Europace, 2019; 21(2): 230-238.

12. GUPTA S, et al. Direct Oral Anticoagulants Versus Vitamin K Antagonists in Patients Undergoing Cardioversion for Atrial Fibrillation: a Systematic Review and Meta-analysis. Cardiovasc Drugs Ther, 2019; 33(3): 339-352.

13. GUTIERREZ C, BLANCHARD DG. Diagnosis and Treatment of Atrial Fibrillation. American Family Physician, 2016; 94(6): 442-452.

14. KHAN AA, LIP GY. The prothrombotic state in atrial fibrillation: pathophysiological and management implications. Cardiovascular Research, 2019; 115(1): 31-45.

15. HINDRICKS G, et al. 2020 ESC Guidelines for the diagnosis and management of atrial fibrillation developed in collaboration with the European Association for Cardio-Thoracic Surgery (EACTS): The Task Force for the diagnosis and management of atrial fibrillation of the European Society of Cardiology (ESC) Developed with the special contribution of the European Heart Rhythm Association (EHRA) of the ESC. European Heart Journal, 2021; 42(5): 373-498.

16. JANUARY CT, et al. 2019 AHA/ACC/HRS Focused Update of the 2014 AHA/ACC/HRS Guideline for the Management of Patients with Atrial Fibrillation: A Report of the American College of Cardiology/American Heart Association Task Force on Clinical Practice Guidelines and the Heart Rhythm Society in Collaboration With the Society of Thoracic Surgeons. Journal of the American College of Cardiology, 2019; 140(2): e125-e151.

17. KHAN AA, LIP GYH. The prothrombotic state in atrial fibrillation: pathophysiological and management implications. Cardiovascular Research, 2019; 115(1): 31-45.

18. KIRCHHOF P, et al. 2016 ESC Guidelines for the Management of Atrial Fibrillation Developed in Collaboration With EACTS. European Heart Journal, 2016; 37: 893-2962.

19. KJEKSHUS VHR, SCHUSTER P. Adherence to oral anticoagulant treatment and risk factor assessment six months after DC-conversion of atrial fibrillation. Scandinavian Cardiovascular Journal, 2020; 54(3): 179-185. 
20. MAHAFFEY KW, TURAKHIA MP. Initiating anticoagulation with the intention of cardioverting: does drug choice matter?. European Heart Journal, 2018; 39(32): 2972-2974.

21. MACHLINE-CARRION MJ. Além do escore CHA2DS2-VASc para predizer o risco de tromboembolismo e acidente vascular cerebral- não é tão simples!. Arquivos Brasileiros de Cardiologia, 2021; 116(2): 332-333.

22. MEAD G, et al. Electrical cardioversion for atrial fibrillation and flutter. Cochrane Database of Systematic Reviews, $2017 ; 11(11)$.

23. MILMAN B, BRUNS BD. Atrial Fibrillation: An Approach to Diagnosis and Management in the Emergency Department. Emergency Medicine Practice, 2021; 23(5): 1-28.

24. RAGO A, et al. Clinical Performance of Apixaban vs. Vitamin $K$ Antagonists in Patients with Atrial Fibrillation Undergoing Direct Electrical Current Cardioversion: A Prospective Propensity Score-Matched Cohort Study. Am J Cardiovasc Drugs, 2019; 19(4): 421-427.

25. RENDA G, et al. Efficacy and Safety of Non-Vitamin K Antagonist Oral Anticoagulants After Cardioversion for Nonvalvular Atrial Fibrillation. The American Journal of Medicine, 2016; 129(10): 1117-1123.

26. ROZEN G, et al. Emergency Department Visits for Atrial Fibrillation in the United States: Trends in Admission Rates and Economic Burden From 2007 to 2014. Journal of the American Heart Association, 2018; 7(15): e009024

27. SOCIEDADE BRASILEIRA DE CARDIOLOGIA (SBC). II Diretrizes Brasileiras de Fibrilação Atrial. Arquivos Brasileiros de Cardiologia, Rio de Janeiro (RJ), 2016; 106(4): supl. 2.

28. SULZGRUBER $P$, NIESSNER A. Changing paradigms in oral anticoagulation during cardioversion in Europe. European Heart Journal- Cardiovascular Pharmacotherapy, 2018; 4(1): 2-3.

29. TRUJILLO TC, et al. Contemporary Management of Direct Oral Anticoagulants During Cardioversion and Ablation for Nonvalvular Atrial Fibrillation. Pharmacotherapy, 2019; 39(1): 94-108. 